\title{
El pueblo, el populismo y otros asuntos
}

\author{
The people, populism and other matters
}

\author{
Lyda Otilia Pérez Acevedo ${ }^{1}$
}

Recibido: Agosto 282014 • Enviado para modificación: Diciembre 42014 • Aceptado: Diciembre 102014.

Pérez, L. (2014). El Pueblo, el populismo y otros asuntos. Revista Ocupación Humana, 14 (2), 37 - 45.

Resumen: El texto fue elaborado por la autora como parte del Seminario Política, ciudadanía y ética pública, del Doctorado en Ciencias Sociales de la Universidad Nacional de Entre Ríos - Argentina. Su propósito es compilar de manera reflexiva aportaciones de diferentes autores respecto de la categoría pueblo y algunas derivaciones que para su comprensión implican al populismo. La autora hace foco en como «el pueblo» puede asumir carácter político en su proceso de nominación y su lugar en las identidades populares. Se hace uso de categorías como espectralidad, hegemonía, heterogeneidad, adversario, entre otras, para profundizar en la complejidad que atañe al tema. Sea este texto una invitación a la comunidad de terapeutas ocupacionales para reflexionar acerca de categorías conceptuales y realidades humanas tan prioritarias y relevantes como es el pueblo y articular de modo crítico dichas nociones en praxis profesionales liberadoras y contra hegemónicas.

Palabras clave: Participación política, participación social, soberanía, hegemonía.

Abstract: The author wrote this manuscript for the 'Seminar on Politics, Citizenship and Public Ethics' in partial fulfillment of her Doctorate degree in Social Sciences at the Universidad Nacional de Entre Ríos in Argentina. Its purpose is to compile, using a reflexive method, the contributions of different authors who have written about "the people" (as a socio-political category) and some derivations thereof that involve understanding populism. The author focuses on how "the people" can both assume its political nature in its nomination process, and its place in their popular identities. The author uses categories such as spectrality, hegemony, heterogeneity, adversary, among others, to deepen the inherent complexity of the topic. May this manuscript be an invitation to the community of occupational therapists to reflect upon conceptual categories and relevant human realities such as "the people;" and to critically articulate these notions in various counter-hegemonic and liberating professional praxis.

Key words: Political participation, social participation, sovereignty, hegemony.

"La democracia es el gobierno por la gente, de la gente, y para la gente" Abraham Lincoln ....y yo me pregunto, ¿quién es "la gente"?

Es común la expresión que en democracia el gobierno es elegido por el pueblo para ejecutar su voluntad, enunciado que se expresa en la Constitución de los distintos países Ilamados democráticos. En el caso de Colombia, desde su Preámbulo se habla del poder soberano del pueblo, no

\footnotetext{
1 Terapeuta Ocupacional. Magíster en Administración de Salud. Candidata a Doctora en Ciencias Sociales. Profesora del Departamento de la Ocupación Humana, Universidad Nacional de Colombia. lopereza@unal. edu.co
} 
obstante acto seguido la Constitución Nacional agrega que dicho poder es representado por sus delegatarios. Vale entonces preguntarse quién/qué es el pueblo, claramente no el que delibera o gobierna, no obstante el principio de la soberanía del mismo, y cuáles son las condiciones de posibilidad y las posibilidades mismas de su soberanía.

De manera cotidiana es posible ilustrar la polisemia del término pueblo, así como su ambigüedad y uso en contextos diversos y hasta antagónicos. Por ejemplo, en julio de 2014 el Presidente de Colombia Juan Manuel Santos expresó su rechazo frente a acciones violentas de la guerrilla y manifestó: "...no podemos seguir indefinidamente en esta situación, porque el pueblo colombiano se confunde y no entiende...el pueblo de Colombia no acepta los ataques a la comunidad ni al medio ambiente... la paciencia del pueblo colombiano no es infinita" (Diario el Universal, 2014); un par de semanas antes, el líder de la guerrilla de las FARC, Timoleón Jiménez, alias Timochenko, cuestionó un discurso del Presidente sobre los diálogos de paz y criticó su énfasis por beneficiar a los propietarios de tierras, inversionistas, Fuerzas Armadas y se preguntó: “...¿¿Y para el pobre pueblo qué? ¿De veras cree Santos que con esas concepciones alcanzará la paz para Colombia?" (Diario el Universo, 2014). Parece ser que en los casos anteriores la noción "pueblo" apunta a comprensiones distintas. ¿Existen entonces tantos pueblos como discursos y formas de comprender las dinámicas sociales y de gobierno de un país?
En el marco de los debates relacionados con la polisemia de la palabra pueblo, me interesan las reflexiones que enfatizan en el carácter político de los procesos de "nominación del pueblo" y sus derivaciones sobre populismo, soberanía, quién lo constituye, quién no, quién o qué es el otro que no es pueblo y cómo se legitiman dichas fronteras.

Un interesante punto de partida proviene de los planteamientos de Laclau (en: Marchart, 2006) respecto de la nominación de un agente social como condición para su existencia, así como la comprensión de la política como el proceso mediante el cual un grupo asume su nombre. Frente a la categoría que aquí interesa, Marchart (2006) distingue "pueblo" como actor de la política o significante dentro del discurso político y "el pueblo" como el sujeto de lo político.

Según Marchart (2006), el nombre "se convierte en el fundamento de la cosa", (pp. 45, 46, 48) pero para desempeñar ese papel tiene que vaciarse de contenido específico para que pueda funcionar como un nombre desprovisto de significados particulares, no obstante refiere más bien a una vaciedad que puedo significar. Entonces este autor plantea que "el pueblo" es el significante vacío o nombre que mantiene unidas identidades populares y que parece convertirse en el nombre para el sujeto político.

Advierte Marchart (2006) que la función representativa del nombre sigue mostrando dos caras: el principioidentidad y la ausente instancia del 
sujeto de lo político dentro del ámbito de lo social.

Con el propósito de explicar el protagonismo de la categoría "pueblo" en el imaginario político occidental, Marchart (2006) encuentra que es debido a su papel central en el dispositivo simbólico de la democracia, dado que ya sin rey y con vaciamiento del lugar del poder, la soberanía no pudo seguir recurriendo a una instancia trascendente de legitimación, de modo que fue ocupada por el nombre del pueblo. Es así que el pueblo tiene que asumir la función clásica de la soberanía, aunque dicha función será precaria, pues el pueblo no puede recurrir a ninguna base trascendental de legitimación. En palabras del autor, el nombre del pueblo asumió la función de una soberanía sin soberano.

Respecto del "pueblo" reconoce Laclau (en: Marchart, 2006) que existe un real del "pueblo" que resiste la integración simbólica. Una posible lectura de esa aseveración sería rastrear la diferencia entre populus (todos los ciudadanos) y plebs (los menesterosos). El pueblo es lo que no puede incluirse en el todo del cual es parte y no puede ser parte del todo en el que está incluido, y hay política porque existe una parte de aquellos que no tienen parte en el todo de una comunidad. Entonces para Laclau el "pueblo" del populismo es una plebs que reivindica ser el único populus legítimo, pero lo heterogéneo ${ }^{2}$ es precisamente lo que queda fuera de este juego, lo que no es parte de la dialéctica entre diferencia y equivalencia, entre la plebs y el populus, lo que escapa a cualquier relación entre parte, no parte y todo.

Por su parte Villavicencio (2010) reconoce que la dualidad contenida en la noción de populus (pueblo) y plebs, el pueblo como soberano y el pueblo como masas pobres, es resuelta por el populismo en la encarnación del todo por la parte. Y es justamente el pueblo excluido, formado por hombres y mujeres humildes, el pueblo corporizado en el sufrimiento o en las fatigas del trabajo, quien viene a ocupar en el populismo el lugar del pueblo soberano. Aquí se puede advertir, a partir de Villavicencio (2010), que la soberanía sí puede ser conquistada siempre que quienes la representan hagan parte de esa parte nunca integrada. Contradicción posible pero por lo menos necesaria de ser alcanzada.

Por su parte Raus (2010) comprende el pueblo como colectividad simbólica de otra idea de justicia, mediación simbólica del nuevo sujeto social que devino del acto de transformación social y de la amplitud del proceso de inclusión. Esta amplitud refiere a una dinámica inclusiva con capacidad de reconfigurar el espacio social y de transformar el ordenamiento entre el sistema de actores y sus particulares relaciones sociales.

Dado que la palabra pueblo es usada para designar el TODO y también para

\footnotetext{
2 Para Marchart (2006, p. 57) lo heterogéneo no puede ser nominado, no tiene un nombre que le pertenezca, no obstante plantea la posibilidad de significarlo desde afuera de una coyuntura hegemónica dada, mediante la estrategia de des-nominar, es decir, de la pluralización de nombres, la pluri-perspectividad de la esfera de la política, donde no se encontrará un nombre único de «el pueblo», sino una pluralidad de nombres.
} 
aludir a la PARTE pobre del pueblo, emerge la tensión todo - parte y sus derivaciones conflicto y consenso y orden y desorden social. Laclau afirma que es de dicha tensión irresuelta de donde emerge el populismo y que tal conflicto está inscrito en su propia naturaleza. Ipola y Portantiero (en: AboyCarlés, 2001) avanzan afirmando que el populismo se caracteriza por la tensión entre ruptura e integración de un mismo espacio político y Aboy-Carlés reconoce que la particularidad del populismo estaría dada por constituir una de las formas de negociar la tensión TODO - PARTE, o en palabras de Laclau, una forma específica de constituir y organizar relaciones sociales. Dicha negociación se gestiona mediante un movimiento pendular que: a) agudiza estas tendencias contrapuestas a través de la alternativa exclusión/inclusión de la alteridad, constitutiva del propio marco de solidaridades; b) afirma y devora alternativamente su propia frontera constitutiva; y c) promueve la emergencia de oposiciones bipolares: uno en su dimensión nacional-popular de ruptura (que los caracteriza en tanto fuerzas reformistas), otro en su dimensión nacional-estatal de integración (que los convierte en provisionales partidos del orden).

La idea dinámica de la frontera constitutiva invita a pensar acerca de quiénes devienen en llamarse pueblo, quiénes no serían pueblo (por lo menos temporalmente) y quiénes los adversarios del pueblo. Lo anterior en el marco de que al existir frontera se construye la categoría adentro-afuera.

Villavicencio (2010) aporta al respecto que en la nueva representación del pueblo por el populismo, emerge un sistema de inclusión/exclusión que impone otros límites infranqueables en el seno de lo social. La distinción patria-antipatria sería un ejemplo. El pueblo como parte -la plebs- ocupa el lugar del todo -el populus-; detrás de esa frontera ya no existe el pueblo, sino el antipueblo. Esa divisoria social, esa frontera insalvable que se instala al interior de lo social, es un elemento constitutivo de la idea de pueblo del populismo.

Al respecto profundiza Aboy-Carlés (en: Barros, 2006) que la exclusión/inclusión del adversario hace a la dinámica del proceso articulatorio e implica una frontera siempre inestable y en desplazamiento constante. En esa movilidad, los adversarios a veces quedan dentro y a veces quedan fuera de la esfera de solidaridades del populismo. Esto constituye fronteras identitarias dinámicas, que se van re-estructurando de forma constante.

Frente a las reflexiones de AboyCarles, Barros (2006) advierte que el populismo supone un conflicto previo a la negociación de la tensión exclusión/inclusión del adversario. Para plantear dicha tensión tienen que haber previamente diferencias en el sistema que son consideradas como adversarias. Entonces argumenta Barros que el populismo es una forma particular de articulación hegemónica en la cual, lo que se pone en juego, es la inclusión radical de una heterogeneidad social respecto del espacio común de representación que supone toda práctica hegemónica. Para incluir o excluir al adversario primero es preciso constituirse como diferencia 
dentro del sistema. Para resistir al otro es necesario antes ser considerado como una diferencia dentro del espacio común de inscripción donde se dan las articulaciones hegemónicas. El populismo es entonces para Barros la radical inclusión de una heterogeneidad que rompe con la homogeneidad institucional. Esa heterogeneidad es la idea de "pueblo" que siempre se resiste a la completa integración simbólica, aún dentro de una articulación populista, y que además distingue el populismo de otro tipo de articulaciones hegemónicas.

La radical inclusión de la heterogeneidad es entendida como ausencia siempre presente que desajusta toda representación. Ausencia porque supone exterioridad respecto del campo de representación como tal, pero siempre presente porque es el suplemento que lo común necesita para poder ser representado. Entonces, frente al argumento sobre el populismo como radical inclusión y puesta en duda de lo común de la comunidad, Barros (2006) concluye que al estar siempre presente como posibilidad lógica es un espectro, y que dicha espectralidad es una amenaza constante a cualquier posibilidad de institucionalización plena.

Al hacer foco en la noción de espectro, agrega Barros que toda articulación hegemónica es asediada por el fantasma de lo excluido y para el caso del populismo la espectralidad se encarna en la figura del pueblo, o en otras palabras, el populismo activa el espectro del pueblo, que aparece y desaparece de la escena remitiendo a esa heterogeneidad excluida siem- pre necesaria (Barros, 2006). Agregan Reano y Yabkowski (2010) que cuando ese espectro asedia, cuando el populismo se activa, se pone en escena esa heterogeneidad excluida; de su carácter espectral proviene entonces la imposibilidad de constituir una institucionalización estable, porque lo que se nos recuerda es que hay algo siempre necesariamente excluido, inaprensible.

Barros retoma a Rancière (en: Barros, 2010) para insistir que el pueblo del populismo es un pueblo que representa una parte que no contaba en la práctica hegemónica vigente, que al no estar representado, no tenía vOz, ni "tomaba parte" y que ahora reclama el todo en nombre de la deuda instaurada por la institucionalidad hegemónica.

Para seguir profundizando en la noción de pueblo son pertinentes las aportaciones de Balsa, a partir de reflexiones de Laclau. Balsa (2010) nombra a la inclusión radical como una drástica ampliación y profundización de la ciudadanía acompañada de una particular lógica para construir una hegemonía de nuevo tipo, donde los excluidos son reconocidos como sujetos colectivos con tradiciones, formas propias de identificación y de ver el mundo, por tanto hace referencia a una inclusión respetuosa de la multiculturalidad; además de propiciar la organización en sus propios colectivos socio-políticos (sindicatos, comunidades, movimientos sociales, etc.) Así mismo esta inclusión instala una discursividad que legitima los beneficios otorgados como derechos inalienables de los ciudadanos en tanto miembros 
de la nación, creando en ellos un sentimiento de activación política y social que modifica el conjunto del orden social (esto implica no solo su relación con el Estado, sino también con la clase dominante y con las capas medias) (Balsa, 2010).

Respecto de la idea de Laclau del populismo como ampliación del campo de lo social y por tanto proceso de construcción de una hegemonía, Balsa (2010) considera más bien que dicha ampliación sería la base necesaria para la hegemonía, dado que la dominación hegemónica plena requeriría de una inclusión previa de todos los potenciales ciudadanos. Continúa Balsa explicando que la lógica populista que da pie a la construcción de una hegemonía peculiar que se constituye por una plebs que reclama ser el único populus legítimo, requiere mantener desplegada una intensa interpelación ideológica de los sectores populares que reactualice permanentemente la ruptura con la oligarquía o grupos dominantes.

Respecto de las clases, Raus (2010) hace un interesante aporte para el caso de Latinomérica, al reconocer que uno de los rasgos sustanciales del populismo latinoamericano es que en el mismo momento que éste expandía objetivamente (económicamente) la clase obrera/ asalariada, lo hacía diluyendo ideológica y políticamente su identidad de clase otorgándole, a través de su interpelación políticodiscursiva, una identidad política popular, de pueblo. Es así que desde la categoría pueblo, confluían todos los que estaban: la clase obrera, el empresariado nacional, el campesino asalariado, el pequeño propietario, el asalariado urbano, el ama de casa, el trabajador autónomo.

En este orden de ideas interesa volver y profundizar en la idea de un "otro" en el pueblo populista. Al respecto Balsa (2010) reconoce que en la lógica populista casi todos los habitantes adultos forman parte de la "ciudadanía" y de la "nación", quedando parcialmente fuera solo "la antipatria", o en otras palabras, los antipopulistas antidemocráticos, es decir aquellos que por un "odio" clasista se oponen a la inclusión radical. Agrega Raus (2010) que ese "otro" antagónico no podía ser una clase ni una fracción de ella, debe ser una identidad con el mismo nivel de agregación (difuso) que el pueblo y con la misma capacidad (cualitativa), y dicha identidad antagónica el populismo la instituyó y fortaleció en la interpelación "oligarquía". Discursivamente el populismo configuró el "otro" político del pueblo en la oligarquía, en quienes estaban fuera del proyecto popular, sea porque este no favorecía sus intereses materiales, sea porque cultural e ideológicamente despreciaban al pueblo y a lo popular.

Por su parte y para el caso de Latinoamérica, Raus (2010) plantea como adversaria de "el pueblo" a la hegemonía conservadora fundada en la organización de los estados nacionales a fines del siglo XIX, sostenida económicamente en el modelo agro o minero exportador en el marco de la división internacional del trabajo, legitimada cultural y socialmente en una matriz social estamental, jerárquica y de muy baja movilidad social. 
Al respecto advierte Balsa (2010) que la inclusión radical es muy disruptiva, difícil de asimilar e implica una redefinición del orden social y de sus jerarquías internas, que cuando se desarrolla y se despliega surgen fuertes sentimientos de rechazo, principalmente desde dos sectores sociales: desde aquellos que sacaban ventaja del sometimiento de los excluidos y desde los sectores medios que antes gozaban de cierto privilegio político y micropoder social sobre los otros sectores hasta entonces no legitimados.

En su intento por recuperar un concepto transformador de "pueblo" y lograr marcar la separación con la oligarquía o el antipueblo, Balsa (2010) propone pensar el pueblo como la suma de las tradiciones populares pasadas por el filtro del "buen sentido" que los explotados suelen contener como sentimiento elemental de separación y de antagonismo frente a los dominantes. Entonces plantea (a partir de Nun, 1989) la importancia de constituir un mutuo esclarecimiento entre las masas explotadas y los intelectuales orgánicos a partir del buen sentido de las mismas.

Respecto de los intelectuales invita Balsa, siguiendo a Gramsci (1929), a que se conecten sentimentalmente con el pueblo-nación de modo que sientan, comprendan, expliquen y justifiquen las pasiones elementales del pueblo. Balsa considera que solo con la delimitación de "lo popular" y una revalorización de la figura de los intelectuales orgánicos como mediadores/ traductores entre las tradiciones populares y la teoría política, es posible evitar los problemas que se derivan del esquema de que "el pueblo nunca se equivoca" (Balsa, 2010) o las críticas acerca de la vaguedad, vacuidad ideológica, antiintelectualismo y carácter transitorio del populismo (Marchart, 2006).

A manera de cierre considero que en la Latinoamérica actual abundan las razones para dinamizar un nuevo sujeto social capaz de transformar y reconfigurar espacios, actores y relaciones sociales, tendientes hacia una mayor justicia y participación. Se han presentado reflexiones de distintos autores que dan cuenta de las condiciones de posibilidad para que "el pueblo", como nombre y realidad social compleja, dinámica, viva, que mantiene identidades populares unidas, sea ese sujeto capaz de encarar y transformar, por ejemplo, situaciones como las que viven en la actualidad Paraguay y Colombia. En el caso de Paraguay, el presidente Horacio Cartes decidió habilitar al ejercito para que intervenga en conflictos internos modificando la Ley de Defensa Nacional (Diario El Clarín, 2013), por su parte el presidente de Colombia, Juan Manuel Santos ordenó la militarización de las principales ciudades el país en el marco de paros campesinos y alteraciones al orden público que incluyen muertes, destrozos y desplazamiento (Diario del Huila, 2013).

En los ejemplos anteriores es posible hacer uso de las categorías perceptuales de figura - fondo para comprender como en situaciones tan difíciles como las que se viven en estas dos naciones, puede "el pueblo" entenderse como la figura que requiere emerger renovada y activa de un fondo complejo (militarización) que contiene multitud 
de elementos interrelacionados (actores y relaciones sociales). No es nueva para la región la constitución de esos "fondos" complejos de donde han emergido "figuras" como la dictadura, las desapariciones y las violaciones a los derechos humanos.

Si las palabras, los nombres, producen existencia, formas de ser y entender el mundo, y si el proceso mediante el cual se asume un nombre corresponde al accionar político, sea el pueblo el nuevo sujeto performativo capaz de dinamizar transformaciones sociales. Que la ausencia siempre presente de lo "heterogéneo" "escurridizo" "excluido" sea el motor perenne que motive la dinámica de "el pueblo". Y en este sentido entonces, ¿Cuáles son las interpelaciones que debemos/podemos hacernos como terapeutas ocupacionales para aportar en la emergencia de "el pueblo"? ¿Nuestra realidad colombiana y regional aboga por una Terapia Ocupacional para y con "el pueblo"? ¿Cómo pueden las universidades de nuestro país privilegiar la formación en un accionar crítico de la Terapia Ocupacional a favor de "el pueblo" y en resistencia del "pueblo"?. Las respuestas están por construirse.

\section{Referencias}

Aboy-Carlés, G. (2001). Repensando el populismo. Ponencia preparada para el XXIII Congreso Internacional Latin American Studies Association Washington D.C. CONICET - Departamento de Política y Gobierno Universidad Nacional de General San Martín. Acceso: 2 jul 2013. Recuperado de: http://lasa.international.pitt.edu/ Lasa2001/AboyCarlesGerardo.pdf.

Balsa, J. (2010). "Las dos lógicas del populismo, su disruptividad y la estrategia socialista", Revista de Ciencias Sociales, segun- da época, año 1, No 17, Bernal, Universidad Nacional de Quilmes.

Barros, S. (2010). Salir del fondo del escenario social. Sobre la heterogeneidad y la especificidad del populismo. Pensamiento Plural. Acceso: 7 agos 2013. Recuperado de: http://pensamentoplural.ufpel.edu.br/ edicoes/05/01.pdf.

Barros, S. (2006). Espectralidad e inestabilidad institucional. Acerca de la ruptura populista. Estudios Sociales, 16(30). Acceso: 5 agos 2013. Recuperado de: http:// www.saap.org.ar/esp/docs-congresos/ congresos-saap/VII/programa/paneles/a/ a2/barros.pdf

Constitución Política de Colombia (1991) Acceso: 23 agos 2014. Recuperado de: http://www.alcaldiabogota.gov.co/sisjur/ normas/Norma1.jsp?i=4125

Diario del Huila (2013). Acceso: 12 oct 2013. Recuperado de: http://www.diariodelhuila.com/site/index.php?option $=$ com_co ntent\&view $=$ article\&id = 39594: santosanuncia-medidas-para-contrarrestar-los12-dias-de-paro-agrario-nacional-\&catid $=29$ : actualidad\&ltemid $=19$.

Diario El Clarín (2013). Acceso: 17 sept 2013. Recuperado de: http://www.clarin. com/mundo/Via-Paraguay-Ejercito-represion-interna_0_979702095.html.

Diario el Universal. (2014). Acceso: 1 sept 2014. Recuperado de: http://www.eluniversal.com.co/colombia/presidente-juanmanuel-santos-endurece-su-discursofrente-al-proceso-de-paz-166274.

Diario el Universo. (2014). Acceso: 1 sept 2014. Recuperado de: http://www. eluniverso.com/noticias/2014/07/22/ nota/3266751/guerrilla-cuestiona-discurso-santos.

Marchart, O. (2006). En el nombre del pueblo. La razón populista y el sujeto de lo político. Cuadernos del CENDES, mayoagosto, 37-58. Universidad Central de Venezuela.

Raus, D. M. (2010). "Situar (una vez más) el debate en torno a la cuestión del popu- 
lismo. Notas y fundamentos", Revista de Ciencias Sociales, segunda época, año 1, No 17, Bernal, Universidad Nacional de Quilmes.

Reano, A. y Yabkowski, N. (2010). "La inestabilidad del demos: repensar la relación entre populismo y democracia", Revista de Ciencias Sociales, segunda época, año
1, No 17, Bernal, Universidad Nacional de Quilmes.

Villavicencio, S. (2010). "El pueblo de la democracia. Forma y contenido de la experiencia populista", Revista de Ciencias Sociales, segunda época, año 1, No 17, Bernal, Universidad Nacional de Quilmes. 